\title{
Modelling and Simulation of the Chemical Homogeneity of the Butadiene-Styrene Copolymer in High-Conversion Emulsion Copolymerization*
}

\author{
Maurycy KALFUs* ${ }^{1}$ and Edward GRZYWA*2 \\ *1 Department of Chemistry, Faculty of Mathematics and Natural Sciences, \\ The Pedagogical College of Slupsk, 76-200 Slupsk, Poland. \\ *2Institute for Organic Industry, 03-236 Warsaw, Poland.
}

(Received November 21, 1977)

\begin{abstract}
The emulsion copolymerization of butadiene with styrene is an example of a commercial process whose selectivity and specificity may be highly improved by technological means feasible now through modelling and simulation, although the basic chemical reactions are neither selective nor specific. To obtain optimum properties of the final rubber, optimum chemical composition is necessary; but since the composition changes during the copolymerization with the conversion of monomers, the resulting copolymer has a heterogeneous chemical composition. This is one of the obstacles to effecting the high-conversion copolymerization. Simulation of the copolymerization under dosing of the more reactive monomer, i. e., butadiene has shown that an improvement in the chemical homogeneity of the copolymer by 17 times at the conversion of $98 \%$ is feasible. A mathematical model accounting for all important effects in the emulsion copolymerization was developed; the most plausible parameters of the model were evaluated by starting from published data; and the simulation was performed by means of programs developed in Fortran for the ICL 2903 computer.

KEY WORDS Mathematical Model / Simulation / Copolymer Chemical Composition / Gel Effect / Dosing of Monomer / Copolymerization Equation Parameters /
\end{abstract}

The importance of selectivity and specificity in chemical reactions as an effective means for optimum utilizing of natural resources and for coping with the pollution problem has become quite evident. This was the first topic of the XXVIth IUPAC Congress held in Tokyo in 1977. For the improvement of the existing large-scale commercial processes, which cannot be immediately replaced by totally new ones, investigations of the processes are still necessary.

The butadiene-styrene rubber comprises an important part of the total synthetic polymer production. The emulsion copolymerization of butadiene with styrene is an example of a commercial process whose selectivity and specificity may be highly improved by technological means, feasible now due to modelling and simulation,

* Presented to the XXVIth IUPAC Congress in Tokyo, September 8, 1977, code 8El 03'. although the basic chemical reactions remain neither selective nor specific.

In order to achieve the optimum properties of the butadiene-styrene rubber, optimum chemical composition, optimum molecular weight distribution, and minimum branching and cross-linking of the copolymer are necessary. ${ }^{1-6}$ All the parameters change with the conversion of monomers, since the chemical reactions during the emulsion copolymerization are neither specific in respect to the chemical composition nor to the size and shape of the resulting macromolecules. This is one of the obstacles in effecting the high-conversion copolymerization. The first problem we tried to solve was the homogeneity of copolymer chemical composition. The approach applied to the solution of this problem seems useful in solving other problems. Thus some of the solutions stated below may prove applicable to other copolymerizations. 
from the simple copolymerization eq 2 with $r_{1}$ and $r_{2}$ only. The last definitions of $C_{\mathrm{crit}}, r_{1}{ }^{\prime}$, and $r_{2}{ }^{\prime}$ do not specify any mechanism involved, and therefore the terms short and entangled have here another meaning than in paper $^{7}$ : they account for all effects at $C>C_{\text {crit }}$ and not only for the gel effect. The limitations of the original eq 1 were recently discussed. ${ }^{8}$ The new eq 1 was assumed in the proposed mathematical model, since for the present state of knowledge ${ }^{1,4,7,8}$ more or less arbitrary assumptions and approximations seem inevitable. $^{7,8}$ Experience in similar modelling ${ }^{2,9-16}$ shows that such models fit the experimental data with a sufficient accuracy for a substantial improvement of commercial processes. A similar approach has proved quite successful. ${ }^{7}$ The approach results in treating the new eq 1 as a semiempirical one; therefore its validity must be evaluated in terms of the agreement of calculated and experimental data. Some problems related to the validity may be discussed theoretically, however.

\section{The Gel and Chain-Transfer Effects}

The mobility of short chains is not strongly affected by diffusional effects, whereas the mobility of chains long enough to become entangled with the neighboring molecules is therefore restricted. The decreased mobility results in a decreased rate of the chain termination by the biradical mechanism, in an increased concentration of growing radicals, and thus in an increased copolymerization rate. A similar effect results from the increased branching of macromolecules due chiefly to chain transfer. ${ }^{1}$ The chain-transfer effect is especially substantial in commercial copolymerizations due to fresh doses of regulators added at higher conversion. ${ }^{2}$ Thus the $C_{\text {crit }}$ value depends on both entanglement and chain-transfer effects.

\section{The Hot-Radicals Effect}

At the start of emulsion polymerization the hotradicals effect $^{8}$ is negligible, since the number of monomer molecules of the order of 100 in micelles $^{1}$ is very small in comparison with the number of molecules around the micelle. Therefore the probability of the dissipation of the hot-radical excess energy before utilizing it in a polymerization reaction is very close to unity. Latex particles are growing during the emulsion copolymerization, reaching in the case of the butadiene-styrene copolymer mean molecular weights corresponding to some 5000 monomer units, ${ }^{2} i . e$., to a number some 50 times higher than the initial number of monomer molecules in the micelles. During the steady-state period at the approximately constant sum of molar concentrations of both monomers, the total number of their molecules increases by some 50 times too. Therefore, with increasing conversion, the above probability may decrease significantly below unity and the hot-radical seffect may not be negligible any more. The effect, if any, will be automatically accounted for when $C_{\text {erit }}, r_{1}{ }^{\prime}$, and $r_{2}{ }^{\prime}$ are fitted appropriately to the experimental data.

\section{The Monomer-Transport Effect}

During the steady-state period of emulsion polymerization the overall rate of polymerization is approximately constant

$$
v=\text { const }
$$

due to the diffusion of monomer molecules from monomer droplets to the latex particles, which will compensate the monomer conversion. According to the Smith and Harkins theory ${ }^{1}$

$$
v=k[\mathrm{M}]_{\mathrm{L}}
$$

where $k$ is a constant and $[\mathrm{M}]_{\mathrm{L}}$ is the monomer concentration in kilomoles per $1 \mathrm{~m}^{3}$ (in SI units) of the latex particles in the emulsion. Therefore eq 3 results in

$$
[M]_{\mathrm{L}}=\left[\mathrm{M}_{1}\right]+\left[\mathrm{M}_{2}\right]=\text { const }
$$

At the start of the copolymerization

$$
\left[\mathrm{M}_{1}\right]_{0} /\left[\mathrm{M}_{2}\right]_{0}=C_{\mathrm{M}_{1}, 0} / C_{\mathbf{M}_{2}, 0}
$$

where the subscript 0 denotes the initial value of $\left[\mathrm{M}_{i}\right]$ and $C_{\mathbf{M}_{i}}$. The monomer $i$ molar concentration $C_{\mathbf{M}_{i}}$ in the whole emulsion in kilomoles per $1 \mathrm{~m}^{3}$ of the emulsion, is the value directly determined by chemical analysis of the emulsion. During the steady-state period of the emulsion copolymerization, the values of $[\mathrm{M}]_{\mathrm{L}}$ do not depend on the polymerization, since the conversion of monomers is compensated by diffusional transport from monomer droplets to latex particles. In the intensively stirred emulsion a state is established which is very close to an equilibrium between the monomers in the latex particles and in the droplets. 


\section{Kalfus and E. Grzywa}

$$
\left[\mathrm{M}_{1}\right] /\left[\mathrm{M}_{2}\right]=C_{\mathrm{M}_{1}} / C_{\mathrm{M}_{2}}
$$

The situation is similar during the last period of emulsion copolymerization, when $v$ drops rapidly to 0 , since there is a partial supplement of the monomer by transport from the droplets. Its rate decreases with increasing conversion, but simultaneously $\left[\mathrm{M}_{i}\right] \rightarrow C_{\mathbf{M}_{i}}$ so eq 7 remains a good approximation. Conversions higher than the one corresponding to the end of the steady-state period may prove economically advantageous in spite of the decreasing $v$, but commercial copolymerization must be stopped at a reasonable $v$ value. Therefore, deviations from eq 7 will have no significant effect on the computed data.

\section{The Model of Copolymerization without Dosing of a Monomer}

This model involves eq 1 and 7 and those which follow. During the copolymerization without dosing of a monomer

$$
C_{\mathbf{M}_{1}, 0}+C_{\mathbf{M}_{2,0}}=C_{\mathbf{M}_{1}}+C_{\mathbf{M}_{2}}+\left(\left[\mathrm{m}_{1}\right]+\left[\mathrm{m}_{2}\right]\right) V_{\mathrm{L}} / V_{\mathrm{E}}
$$

where $V_{\mathrm{L}}$ and $V_{\mathrm{E}}$ are the total volumes of the latex particles and of the emulsion, respectively, and thus $V_{\mathrm{L}} / V_{\mathrm{E}}$ is the volume fraction of latex particles in the emulsion-a factor necessary for the comparison of $C_{\mathbf{M}_{i}}$ with $\left[\mathrm{m}_{i}\right]$ in agreement with their definitions. Since $\left[\mathrm{m}_{i}\right]$ increases during the copolymerization, a decrease in $C_{\mathbf{M}_{i}}$ results from eq 8 even during the steady-state emulsion copolymerization period, when eq 5 holds. The decrease in $C_{\mathbf{M}_{i}}$ results in changes in $\left[\mathrm{M}_{i}\right]$ according to eq 7 , which may be rewritten as

$$
\left[\mathrm{M}_{1}\right]=C_{\mathrm{M}_{1}}\left[\mathrm{M}_{2}\right] / C_{\mathrm{M}_{2}}=C_{\mathrm{M}_{1}} f\left(C_{\mathrm{M}_{2}}\right)
$$

where $f\left(C_{\mathbf{M}_{2}}\right)$ is a function of the decreasing $C_{\mathbf{M}_{2}}$. With decreasing $\left[\mathrm{M}_{1}\right]$, i.e., with the decreasing concentration of the more reactive monomer in latex particles, $\left[\mathrm{M}_{2}\right]$ will increase according to eq 5 in spite of the decreasing of $C_{\mathbf{M}_{2}}$, which proceeds simultaneously with a decrease in $C_{\mathbf{M}_{1}}$. The solution of the system of eq $1,5,8$, and 9 is rather difficult, but eq 5 refers to the copolymerization kinetics only and has no effect on the copolymer chemical composition: the last is described by eq 1 , where only $x \equiv\left[\mathrm{M}_{1}\right] /\left[\mathrm{M}_{2}\right]$ is needed, and therefore eq 7 or 9 may be used in the model without eq 5 for replacing the practically inaccessible $\left[\mathrm{M}_{i}\right]$ by $C_{\mathbf{M}_{i}}$ which is experimentally easy to determine. Thus, the following equations with $\left[\mathrm{M}_{i}\right]$ related to
$C_{\mathrm{M}_{i}}$ by eq 7 or 9 were developed and included into the model

$$
R_{n}=R\left(x_{n}\right), x_{n} \equiv M_{1 n} / M_{2 n}=C_{\mathbf{M}_{1, n}} / C_{\mathbf{M}_{2, n}}
$$

where $n$ denotes the $n$-th conversion step, modelled by the $n$-th iteration step in the simulation process. Equation 10 shows that for a given set of the four relative reactivities, $r$, in eq 1 , the value of the three equal ratios in eq 1 becomes a function only of the variable $x_{n}$. The function $R\left(x_{n}\right)$ changes at $n_{\text {crit }}$, corresponding to $C_{\mathrm{cr} 1 \mathrm{t}}$, when $r_{i}{ }^{\prime}=r_{i}$ no longer holds.

In the accepted model at each $n$-th step

$$
\begin{gathered}
\Delta\left[\mathrm{m}_{2}\right] V_{\mathrm{L}} / V_{\mathrm{E}}=\Delta C_{\mathbf{M}_{2}}=p C_{\mathbf{M}_{2,0}}=\text { const } \\
C_{\mathbf{M}_{2, n}}=C_{\mathbf{M}_{2,0}}-n \Delta C_{\mathbf{M}_{2}}
\end{gathered}
$$

and

$$
\left[\mathrm{m}_{2}\right]_{n} V_{\mathrm{L}} / V_{\mathrm{E}}=n \Delta C_{\mathrm{M}_{2}}
$$

where $p$ is an arbitrary fraction constant during a set of simulation runs. In the simulation runs stated below $p=0.001$ was assumed, although at $p=0.01$ similar data was obtained.

Equations 1, 10, and 11 result in

$$
\begin{gathered}
\Delta\left[\mathrm{m}_{1}\right]_{n} V_{\mathrm{L}} / V_{\mathrm{E}}=\left(\Delta C_{\mathrm{M}_{1}}\right)_{n}=R_{n} \Delta C_{\mathbf{M}_{2}} \\
C_{\mathbf{M}_{1, n}}=C_{\mathbf{M}_{1}, 0}-\sum_{j=1}^{n}\left(\Delta C_{\mathrm{M}_{1}}\right)_{j} \\
{\left[\mathrm{~m}_{1}\right]_{n} V_{\mathrm{L}} / V_{\mathbf{E}}=\sum_{j=1}^{n}\left(\Delta C_{\mathbf{M}_{1}}\right)_{j}}
\end{gathered}
$$

where the symbol of difference, $\Delta$, replaces the symbol of differential, $d$, in eq 1 .

Equation 11 shows that, in the accepted model, changes in the concentration of bound units and free molecules of the less reactive monomer 2 are constant and equal to a constant part of the initial concentration of the monomer 2 , and therefore concentrations in eq 12 and 13 are simple functions of $n$. These constant increments of course, refer not necessarily to constant time intervals: the time intervals, $(\Delta t)_{j}$, and the reaction rates, $\left(\Delta\left[\mathrm{m}_{2}\right]_{j} /\right.$ $(\Delta t)_{j}=\left(\Delta\left[\mathrm{M}_{2}\right]_{j} /(\Delta t)_{j}\right.$, corresponding to the copolymerization and iteration $j$-th steps, may both change during the copolymerization, but this problem is beyond the scope of the proposed model.

The Model of Copolymerization with Dosing of the More Reactive Monomer

Runs with dosing of the more reactive monomer 
1 , in this case, butadiene, were simulated in order to evaluate the efficiency of the dosing in maintaining the optimum chemical composition of the copolymer during the whole period of copolymerization.

Since, due to the dosing, the total amounts of monomer 1 changed during the copolymerization, the conversion referred in these runs to monomer 2 and is defined as

$$
c_{2}=\left[\mathrm{m}_{2}\right] V_{\mathrm{L}} / V_{\mathrm{L}} C_{\mathbf{M}_{2,0}}
$$

and the concentration of copolymer in micelle particles is given as a molar ratio.

$$
C=\left(\left[\mathrm{m}_{1}\right]+\left[\mathrm{m}_{2}\right]\right) /\left(\left[\mathrm{m}_{1}\right]+\left[\mathrm{m}_{2}\right]+\left[\mathrm{M}_{1}\right]+\left[\mathrm{M}_{2}\right]\right)
$$

The optimum initial composition of the monomer mixture was computed as the initial molar ratio of the more and the less reactive monomers, $X_{0}$, from eq 20

$$
R_{0}=M_{2}\left(100-P_{\mathrm{opt}}\right) / P_{\mathrm{opt}} M_{1}
$$

and

$$
X_{0}=\left\{\left[\left(1-R_{0}\right)^{2}+4 R_{0} r_{1} r_{2}\right]^{1 / 2}-1+R_{0}\right\} / 2 r_{1}
$$

where $R_{0}$ is the initial value of $R, M_{i}$ is the molecular weight of monomer $i$, and $P_{\text {opt }}$ is the optimum content of the bound less reactive monomer 2 in the copolymer, expressed in percents based on weight. Since, at the start of the copolymerization, $r_{i}{ }^{\prime}=r_{i}$, the computation of $X_{0}$ from eq 20 involves the input of $M_{1}, M_{2}, P_{\text {opt }}$, and $r_{1}$ and $r_{2}$ only.

The rate of dosing is defined as

$$
Y_{g, h}=\left(\Delta C_{\mathbf{M}_{1}}\right)_{g, h} /(\Delta t)_{h} \quad g=1,2
$$

Its value is computed by two different methods for each $h$-th period of dosing

$$
\left(\Delta C_{\mathbf{M}_{1}}\right)_{1, h}=X_{0} C_{\mathbf{M}_{2, h}}-C_{\mathbf{M}_{1, h}}
$$

and

$$
\left(\Delta C_{\mathbf{M}_{1}}\right)_{2, h}=2\left(\Delta C_{\mathbf{M}_{1}}\right)_{1, h}
$$

The values which result from eq 22 correspond to $X_{0}$ being restored at the start of each $h$-th period of dosing of monomer 1 , whereas the values which result from eq 23 correspond to $X_{0}$ being restored at the medium moment of each period, $i . e$, between the moments of dosing. The effect of dosing is accounted for by

$$
C_{\mathbf{M}_{1, n}}=C_{\mathbb{M}_{1, n-1}}-\left(\Delta C_{\mathbb{M}_{1}}\right)_{n}+\left(\Delta C_{\mathbf{M}_{1}}\right)_{g, h}
$$

During the simulation of continuous dosing $g=1$ and $h=n$, whereas during the periodical dosing $h=n / q$, where $q$ is a natural number defining the periods between dosing, $(\Delta t)_{h}$, in terms of the corresponding periods, $(\Delta t)_{j}$, between the copolymerization or iteration steps.

$$
(\Delta t)_{h}=\sum_{j=q h-q+1}^{q h}(\Delta t)_{j}
$$

Thus, eq 19-25 describe 3 different methods of dosing of the more reactive monomer: the continuous method and two different periodical

\begin{tabular}{|c|c|c|c|}
\hline Ref no. & $r_{1}$ & $r_{2}$ & Authors \\
\hline & 1.20 & 0.60 & \\
\hline & 1.40 & 0.50 & Meehan E. J., 1946 \\
\hline & 1.60 & 0.40 & \\
\hline 1 & 1.39 & 0.78 & Lewis F. M., 1948 \\
\hline \multirow[t]{3}{*}{2} & 1.48 & 0.23 & \multirow{3}{*}{ Henry-Logan K. R., } \\
\hline & 1.35 & 0.58 & \\
\hline & 1.59 & 0.44 & \\
\hline $3 a$ & 1.40 & 0.70 & \multirow{6}{*}{ Mitchell J. M., 1949} \\
\hline 3 & 1.80 & 0.60 & \\
\hline \multirow[t]{4}{*}{$3 b$} & 2.20 & 0.50 & \\
\hline & 1.51 & 0.81 & \\
\hline & 1.83 & 0.65 & \\
\hline & 2.15 & 0.49 & \\
\hline 5 & 1.38 & 0.64 & \multirow{3}{*}{ Gilbert R. D., 1952} \\
\hline \multirow[t]{2}{*}{6} & 1.37 & 0.38 & \\
\hline & 1.83 & 0.65 & \\
\hline $8 \mathrm{a}$ & 1.20 & 0.02 & \multirow{3}{*}{ Ashikari N., 1959} \\
\hline 8 & 1.30 & 0.01 & \\
\hline $8 \mathrm{~b}$ & 1.40 & 0.00 & \\
\hline 4 & 1.40 & 0.38 & \multirow{2}{*}{ Orr R. J., 1961} \\
\hline 7 & 1.40 & 0.44 & \\
\hline
\end{tabular}
methods.

Starting from the models, programs in Fortran IV for the ICL 2903 computer were developed.

\section{EXAMINATION OF PUBLISHED RELATIVE REACTIVITIES}

The $r_{1}$ and $r_{2}$ values published for the butadiene-styrene system are stated in Table II.

Table II. Examined $r_{1}$ and $r_{2}$ values

The values differ markedly even for emulsion copolymerizations under the same conditions. In cases when the authors have stated the ranges of the values, they were rather broad; this is especially evident when minimum $r_{1}$ values are combined 


\section{Kalfus and E. Grzywa}

with maximum $r_{2}$ values (first lines in each set of 3 lines in Table II) and vice versa (last lines in each set). The effect of the discrepancies on the copolymer composition is quite substantial, as seen in Figures 1 and 2 from the graphs of styrene content in the copolymer in percents based on weight, $P, v s$. the mean conversion, $c$, defined as the molar ratio:

$$
c=\frac{\left(\left[\mathrm{m}_{1}\right]+\left[\mathrm{m}_{2}\right]\right) V_{\mathrm{L}} / V_{\mathrm{E}}}{C_{\mathbf{M}_{1,0}}+C_{\mathbf{M}_{2,0}}}=\frac{C_{\mathbf{M}_{1,0}}+C_{\mathbf{M}_{2,0}}-\left(C_{\mathbf{M}_{1}}+C_{\mathbf{M}_{2}}\right)}{C_{\mathbf{M}_{1,0}}+C_{\mathbf{M}_{2,0}}}
$$

The $P$ values as a function of $c$ were computed from eq 2 by the above mentioned program.

In Figure 1 the curves are stated for mean $r_{1}$ and $r_{2}$ values, denoted by the same reference numbers in Figure 1 and in Table II, whereas in Figure 2 they were stated for the $r_{1}$ and $r_{2}$ ranges

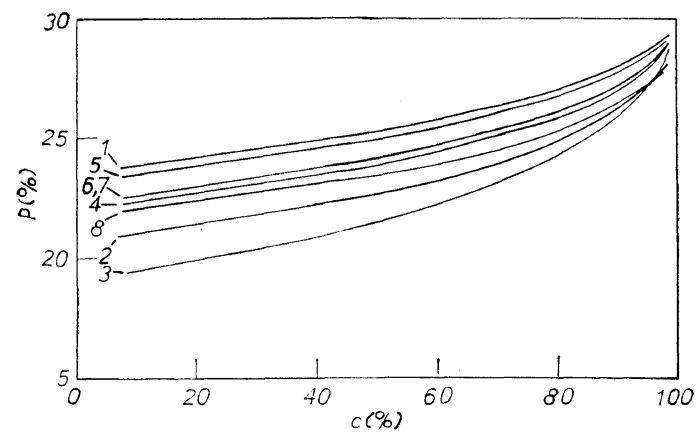

Figure 1. Styrene contents, $P$, vs. conversion, $c$, for mean published $r_{1}$ and $r_{2}$ values.

Curves are numbered according to ref no. in Table II, column 1 .

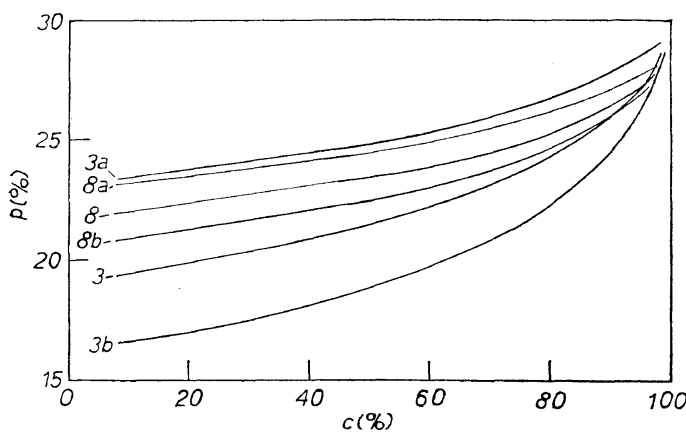

Figure 2. Styrene contents, $P$, vs. conversion, $c$, for published $r_{1}$ and $r_{2}$ ranges.

Curves are numbered according to ref no. in Table II, column 1. published by some authors and denoted in a similar way. In view of the discrepancies a more sophisticated model than the one proposed seems premature.

\section{SELECTION OF \\ THE COPOLYMERIZATION-EQUATION PARAMETERS}

Since eq 1 is a semiempirical one, no theoretical considerations or independent measurements yield the appropriate $C_{\mathrm{crit}}, r_{1}{ }^{\prime}$, and $r_{2}{ }^{\prime}$ values; they must therefore be evaluated or selected by best fit of the resulting curves to experimental data. Examination of data computed for 120 sets of the 3 parameters has shown that the most plausible $C_{\text {crit }}$ values are in the ranges of $0.2-0.6$, and that changes in $C_{\text {erit }}$ over 0.6 have no substantial effect on the chemical composition of the copolymer. Some of the data computed for $r_{1}=1.40$ and $r_{2}=0.38$ and for the initial monomer mixture containing $30-\%$ styrene based on weight are stated in Figure 3. The effect of $r_{1}{ }^{\prime}$ and $r_{2}{ }^{\prime}$ on the content of styrene in copolymer, $P$, was similar for other $r_{1}$ and $r_{2}{ }^{\prime}$ values and for the initial styrene content in the monomer mixture, $P_{0}$, equal to $25 \%$.

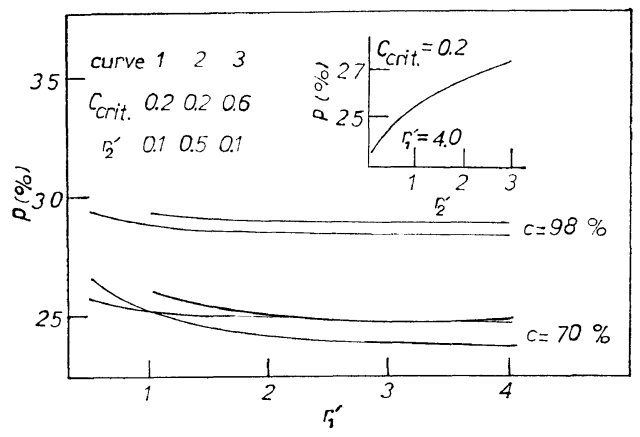

Figure 3. Examples of styrene content, $P$, as function of $C_{\mathrm{crit}}, r_{1}{ }^{\prime}$, and $r_{2}{ }^{\prime}$.

As shown in Figure 3, a significant effect was observed for $r_{1}{ }^{\prime}<1.5$ and $r_{2}{ }^{\prime}<3$ only. The observations were accounted for in the selection of the 3 parameters, i.e., the checked sets of the 3 parameters covered the above ranges.

The developed selection procedure was based on the least-squares method. The procedure involved computing of the styrene contents in copolymer, 
$P_{y}$, for a series of mean conversions, $c_{y}$, corresponding to published data on the styrene content, $\boldsymbol{P}_{\boldsymbol{e}, y}$, for each checked set of the 3 parameters. The computed styrene content in the increments, $\mathrm{d} \boldsymbol{P}_{z}$, bound in the copolymer at a moment, and the respective published data, $\mathrm{d} P_{e, z}$, were also used in the selection procedure.

The sums, $Q$, of the squares of the differences in the computed and published data were computed

$$
Q=\sum_{y=1}^{u}\left(P_{y}-P_{e, y}\right)^{2}+\sum_{z=1}^{w}\left(\mathrm{~d} P_{z}-\mathrm{d} P_{e, z}\right)^{2}
$$

where $u$ and $w$ were the numbers of the experimental pairs $c_{y}-\boldsymbol{P}_{e, y}$ and $c_{z}-\mathrm{d} \boldsymbol{P}_{e, z}$, respectively, used as the reference ones in the selection procedure. The selection method included an accounting for the different reliability of the reference data by the corresponding stepwise excluding of the less reliable ones from the summation in eq 27, yielding thus different types of $Q$ values.

The procedure is explained by the example of its tentative application, as stated in Tables III and IV.

In Table III the $P_{e}$ and $\mathrm{d} P_{e}$ values, used tentatively as the reference ones are stated for two

Table III. Reference composition data

\begin{tabular}{|c|c|c|c|c|}
\hline \multirow{2}{*}{$\begin{array}{l}\% \text { based } \\
\text { on weight }\end{array}$} & \multirow{2}{*}{$P_{0}$} & \multicolumn{3}{|c|}{$C$} \\
\hline & & 5 & 70 & 98 \\
\hline \multirow{2}{*}{$P_{e, y}$} & 30 & 21.5 & 25.0 & 30.0 \\
\hline & 25 & 17.1 & 20.5 & 25.0 \\
\hline \multirow{2}{*}{$\mathrm{d} P_{e, z}$} & 30 & - & 31.0 & - \\
\hline & 25 & - & 26.5 & - \\
\hline
\end{tabular}

initial contents of styrene in the monomers mixture, $P_{0}$. The $P_{e, y}$ and $\mathrm{d} P_{e, z}$ values for $c_{e}=5 \%$ and $c_{e}=70 \%$ were evaluated, starting from the published data, ${ }^{17,18}$ whereas the $P_{e, y}$ values for $c_{e}=$ $98 \%$ were assumed to be equal to the theoretical ones at $c=100 \%$ and to coincide therefore with the $P_{0}$ values. In Table IV the best fit values are stated for two pairs of $r_{1}$ and $r_{2}$ values, selected at $r_{1}{ }^{\prime}=$ $r_{1}$ and $r_{2}{ }^{\prime}=r_{2}$ by the above procedure from the 21 pairs stated in Table II. The checked $C_{\text {crit }}$ values $0.2,0.4$, and 0.6 cover the ranges which result from Figure 3, whereas $C_{\text {erit }}=1.0$ means just that $r_{i}{ }^{\prime}=$ $r_{i}$ during the whole copolymerization. The checked $r_{2}{ }^{\prime}$ values cover the above ranges, but the $r_{1}{ }^{\prime}$ values were checked over wider ranges, $i . e$. , up to 4.0. Combination of the checked values of the three parameters yields 105 sets of the three parameters for each of the two pairs of $r_{1}$ and $r_{2}$ values under the limiting condition of $r_{1}{ }^{\prime} \geq r_{2}{ }^{\prime}$, which results from the higher relative reactivity of butadiene in the butadiene-styrene system. In the tentative application of the selection procedure three kinds of $Q$ of different reliability, i.e., $Q_{8}, Q_{6}$, and $Q_{4}$, were assumed, referring to all 8 values in Table III, to all 6 values of $P_{e, y}$ and to 4 values of $P_{e, y}$ at $c_{e}=5 \%$ and $c_{e}=70 \%$, respectively. In all cases the $Q$ values for $C_{\text {erit }} \leq 0.6$ are lower than for $C_{\text {crit }}=1.0, i$.e., the curves from the introduced $r_{1}{ }^{\prime}$ and $r_{2}{ }^{\prime}$ values fitted the reference data better. The maximum difference of $41 \%$ was found for $Q_{8}$ equal to 7.22 and 5.11. The minimum $Q_{4}, Q_{6}$, and $Q_{8}$ values, corresponding to best fit values of the checked parameters, are stressed for both $r_{1}$ and $r_{2}$ pairs in Table IV. Here the $Q$ values next to them in their low values are stated

Table IV. Best fit values by the LSQ method ${ }^{\mathrm{a}}$

\begin{tabular}{|c|c|c|c|c|c|c|c|c|c|c|c|}
\hline \multicolumn{6}{|c|}{$r_{1}=1.40, r_{2}=0.38$} & \multicolumn{6}{|c|}{$r_{1}=1.38, r_{2}=0.64$} \\
\hline$r_{1}^{\prime}$ & $r_{2}^{\prime}$ & $C_{\text {crit }}$ & $Q_{4}$ & $Q_{6}$ & $Q_{8}$ & $r_{1}^{\prime}$ & $r_{2}^{\prime}$ & $C_{\text {crit }}$ & $Q_{4}$ & $Q_{6}$ & $Q_{8}$ \\
\hline - & - & 1.0 & 3.27 & 4.95 & 7.22 & - & - & 1.0 & 11.20 & 12.29 & 14.74 \\
\hline 1.0 & 0.5 & 0.6 & 3.73 & 4.70 & 5.34 & 1.0 & 0.1 & 0.2 & 9.82 & 11.80 & 15.65 \\
\hline 1.5 & 0.2 & 0.4 & 2.96 & 5.60 & 11.34 & 1.0 & 0.2 & 0.2 & 10.41 & 11.87 & 14.48 \\
\hline 2.0 & 1.0 & 0.6 & 3.67 & 4.71 & 5.17 & 1.5 & 0.2 & 0.2 & 9.41 & 11.72 & 16.94 \\
\hline 2.5 & 1.0 & 0.6 & 3.54 & 4.76 & 5.11 & 1.5 & 0.5 & 0.2 & 10.68 & 11.89 & 13.98 \\
\hline 4.0 & 1.0 & 0.6 & 3.35 & 4.91 & 6.15 & 2.0 & 0.5 & 0.2 & 9.91 & 11.45 & 14.60 \\
\hline
\end{tabular}

a The examined values $\left(r_{1}{ }^{\prime} \geq r_{2}{ }^{\prime}\right): 105$ combinations per a $r_{1}, r_{2}$ pair

$r_{1}^{\prime}: 0.5,1.0,1.5,2.0,2.5,3.0,3.5,4.0$.

$r_{2}^{\prime}: 0.1,0.2,0.5,1.0,2.0,3.0$.

$C_{\text {crit }}: 1.0,0.6,0.4,0.2$. 


\section{Kalfus and E. GRzYwA}

for comparison of the changes in $Q_{4}, Q_{6}$, and $Q_{8}$ values within each set of the checked parameters. The comparison shows the importance of the estimation of the reliability of the reference data for the final selection of the three parameters.

\section{EFFECT OF DOSING OF THE MORE REACTIVE MONOMER}

The effect of the dosing is evaluated by comparison of the copolymerization without dosage of butadiene with that proceeding under continuous or periodical dosage. The two different methods specified in eq 22 and 23 were used to study the periodical dosage. The compared data are stated in Table $\mathrm{V}$ for the five sets of the most plausible values of $r_{1}, r_{2}, r_{1}{ }^{\prime}, r_{2}{ }^{\prime}$, and $C_{\text {crit }}$ selected from those stated in Table IV.

Table V. Maximum difference, $\Delta P$, and maximum deviation, $\delta P$, at optimum parameters in eq 1

\begin{tabular}{|c|c|c|c|c|c|c|}
\hline$r_{1}$ & & 1.4 & 1.4 & 1.4 & 1.38 & 1.38 \\
\hline$r_{2}$ & & 0.38 & 0.38 & 0.38 & 0.64 & 0.64 \\
\hline$r_{1}^{\prime}$ & & 1.0 & 1.5 & 2.5 & 1.5 & 2.0 \\
\hline$r_{2}^{\prime}$ & & 0.5 & 0.2 & 1.0 & 0.5 & 0.5 \\
\hline$C_{\text {crit }}$ & & 0.6 & 0.4 & 0.6 & 0.2 & 0.2 \\
\hline No dosage & $\int \Delta P$ & 6.86 & 6.36 & 6.76 & 5.65 & 5.54 \\
\hline$P_{0}=30 \%$ & $\delta \delta P$ & 4.27 & 3.77 & 4.17 & 4.19 & 4.08 \\
\hline Periodical & $\int \Delta P$ & 0.99 & 0.38 & 0.80 & 0.41 & 0.82 \\
\hline dosage 1 & $\{\delta P$ & 1.21 & 0.25 & 1.02 & 0.22 & 0.63 \\
\hline Periodical & $\int \Delta P$ & 0.71 & 0.79 & 0.49 & 0.70 & 1.16 \\
\hline dosage 2 & $\delta P$ & 0.71 & 0.57 & 0.49 & 0.52 & 0.98 \\
\hline Continuous & $\int \Delta P$ & 0.65 & 0.64 & 0.42 & 0.60 & 1.07 \\
\hline dosage & $\delta P$ & 0.65 & 0.64 & 0.42 & 0.60 & 1.07 \\
\hline
\end{tabular}

The maximum difference in the styrene content, $\Delta P, i . e$. , the difference between the maximum and minimum styrene content in the copolymer observed in each of the simulated runs up to $c=$ $98 \%$, and the absolute maximum deviation, $\delta P$, of the styrene content in the copolymer from its assumed optimum content of $25.0 \%$ were used as criterions for the comparison. Both criterions depend significantly on the above five parameters, but for all the five most plausible sets stated in Table $\mathrm{V}$ an important improvement in the chemical homogeneity of the copolymer was found: the maximum improvement corresponds to the de- crease in $\triangle P$ and $\delta P$ during the periodical dosage 1 by 17 and 15 times, respectively (column 2), and the minimum improvement, corresponding to the decrease by 5 and 4 times, respectively, was found in a run with continuous dosage (column 5). Thus, the data in Table $\mathrm{V}$ show that a very substantial improvement in the chemical homogeneity of copolymer is feasible by technological means in high-conversion copolymerization.

Acknowledgment. We thank to Dr. Kazuyuki Horie of The University of Tokyo for the fruitful discussion on our study and especially on eq 5,7 , 10-16, and 24.

\section{REFERENCES}

1. H. G. Elias, "Makromolekule," Huthig-Wepf Verlag, Basel-Heidelberg, 1972.

2. M. Kalfus and J. Kopytowski, et al., Vysokomol. Soedin., 7, 1655 (1965).

3. E. Grzywa and E. Krotki, Polimery, 9, 374 (1964).

4. E. Krotki, "Investigations in Emulsion Polymerization of Butadiene and Copolymerization of Butadiene with Styrene at High Conversion of Monomers (in Polish), Chemical Works "Oswiecim," Oswiecim, Poland, 1974; a Doctor Thesis presented at the Silezian University, Katowice, Poland.

5. T. J. Alfrey, J. J. Bohrer, and H. Mark, "Copolymerization," Interscience Publishers, New York, N. Y., 1952.

6. G. E. Ham, "Copolymerization," Interscience Publishers, New York, N. Y., 1964.

7. J. N. Cardens and K. F. O'Driscoll, J. Polym. Sci., Polym. Chem. Ed., 14, 883 (1976).

8. E. Tudos, T. Kelen, and T. Foldes-Berezhnikh, J. Polym. Sci., Polym. Symp. Ed., No. 50, 109 (1975).

9. M. Kalfus, Annales Soc. Chem. Polym. (in English), 39, 309 (1965).

10. M. Kalfus, Makromol. Chem., 83, 287 (1965).

11. M. Kalfus and J. Mitus, J. Polym. Sci. Part, A-1, 4, 953 (1966).

12. M. Kalfus and H. G. Elias, J. Macromol. Sci. Chem., A1, 955 (1967).

13. H. G. Elias and M. Kalfus, Makromol. Chem., 105, 95 (1967).

14. P. Szewczyk and M. Kalfus, J. Macromol. Sci. Chem., A7, 737 (1973).

15. M. Kalfus and B. Dudek, Makromol. Chem., 178, 1609 (1977)

16. M. Kalfus and A. Jurkiewicz, et al., "Abstracts of Scientific Papers, XXth IUPAC Congress," Moscow, 1965, 3, 166 (Abstract No. F 52).

17. E. J. Meehan, Rubber Chem. Technol., 20, 14 (1947).

18. R. L. Maier, Ind. Eng. Chem., 41, 1570 (1949). 